\title{
Patterns detection in diffraction images of transmission electron microscopy
}

\author{
S.G. Nebaba, A.Ya. Pak \\ stepan-lfx@mail.ru|ayapak@tpu.ru \\ Tomsk Polytechnic University, Tomsk, Russia
}

\begin{abstract}
Specialized software that supports existing approaches to processing images of the crystal structure of materials for analyzing transmission electron microscopy images have a lot of different digital image processing methods, but major part of it are weakly automated. In some tasks automated algorithms of image processing have been developed, e.g. in task of estimation of the width of a layer of material from a raster image. The paper considers the problem of automated processing of diffraction images obtained by transmission electron microscopy. A number of modifications, such as Watershed algorithm, binarization and Fast Fourier Transform, are proposed for existing image processing algorithms. These modifications can help automate the processing of the diffraction pattern of a material sample from an image of transmission electron microscopy. The given examples of image processing of particular cases of diffraction patterns have shown the prospects for the development of algorithm based on combination of the proposed modifications of considered algorithms. Adaptive binarization with Watershed segmentation would be useful in automated distance estimation in transmission electron microscopy images.
\end{abstract}

Keywords: computer vision, image processing, image analysis, transmission electron microscopy, crystalline diffraction pattern.

\section{Introduction}

Methods and algorithms of computer vision associated with the processing and analysis of raster images are widely used in various fields of science [1-5], including in the field of processing images obtained using electron microscopes [6]. One of the fundamental problems in these areas is the automation of assessing the composition and structure of materials from their images. An effective solution of these problems simplifies the tasks of nondestructive quality control of materials and products, their identification and determination of their properties and appearance [6].

Transmission electron microscopy (TEM) involves the study of thin images using a beam of electrons passing through them and interacting with them. The electrons that pass through the sample are focused on the imaging device: a fluorescent screen, a photographic plate or a camera sensor. Using TEM, it is possible to study objects even at the atomic level. At relatively low magnifications, the contrast on the TEM arises due to the absorption of electrons by the material of the test sample. At high magnifications, the complex interaction of waves forms an image that requires a more complex interpretation. Modern TEMs have operating modes that allow one to study the elemental composition of samples, crystal orientation, phase shift of electrons, etc.

The TEM method is used to assess the structure of the material, both in the volume of the sample and in its surface region. TEM is one of the most highly informative research methods used in materials science, solid state physics, biology, and other sciences.

The ability to observe the electron diffraction pattern (or, in other words, to observe cross sections of the reciprocal lattice of the sample) simultaneously with the image of the microstructure of the sample makes it possible to obtain valuable information on the symmetry of the crystal lattice and structural defects of the material under study. Comparison of microphotography with electron diffraction patterns makes it possible to correlate the microstructure elements with a particular crystalline or amorphous phase identified on the electron diffraction pattern. The TEM method can be applied to solve problems such as:

- characterization of the structure of the sample in volume and on the surface;

- determination of the qualitative phase composition of the sample;

- determination of orientational relations between the elements of the structure of the sample.

The development of information technology and computing devices contributes to progress in solving such problems. However, nowadays, existing methods for identifying and evaluating microobjects from a raster image do not have sufficient universality that would make it easy to automate them. In addition, these methods and algorithms are often part of proprietary software that is closely associated with electronic microscopy equipment and is protected by the copyright of the manufacturers of this equipment [7]. The cost of such equipment can be high, which is unacceptable for a fairly significant part of researchers and scientific organizations.

At the same time, systems for analyzing such images can be widely demanded by many scientific organizations as well as enterprises in the manufacturing sector, making it possible to carry out operational control and analysis of the composition and structure of materials and products. Cheap analogues of existing software for critical areas of enterprise activity can stimulate the development of technologies for the synthesis, analysis and production of micro- and nanomaterials. Ultimately, improving the characteristics of materials for various purposes and products based on them can both have a positive impact on the level of safety in many areas of human activity, and contribute to the development of new technologies.

In [6] and many others papers, the practical use of specialized software for processing images obtained using an electron microscope, can be seen. That indicates the development of this field of knowledge in the modern world.

Thus, the interdisciplinary topic under consideration seems relevant and promising. There is a need to systematize existing methods and algorithms for the automated evaluation and processing of images and to 
develop software for the evaluation and analysis of images of electron microscopy based on them. In the future, this could contribute to the development of software systems for analyzing images of electron microscopes with the possibility of mass application and implementation.

\section{Automation of the algorithm for estimating the width of a layer of material from a raster image}

Earlier in [8], an automated algorithm was proposed for estimating the width of material layers from a raster image. The key idea of it is to select, normalize, and evaluate an area with a regular layered structure of a raster image obtained using an electron microscope. Also, affine transformations were used in the algorithm in order to build a regular structure strictly perpendicular to the abscissa axis and simplify further calculations [9], binarization [4] and the calculation of derivatives of the obtained one-dimensional sequences.

With the known relation between the image points and the actual physical quantities, it is possible to obtain the layer width estimated by the physical quantity.

The proposed algorithm is quite simple from the point of view of implementation, and does not require large computational resources.

The described algorithm was tested on several images shoot in direct resolution mode; crystalline planes in the composition of the crystalline material are visible in such images (figure 1).

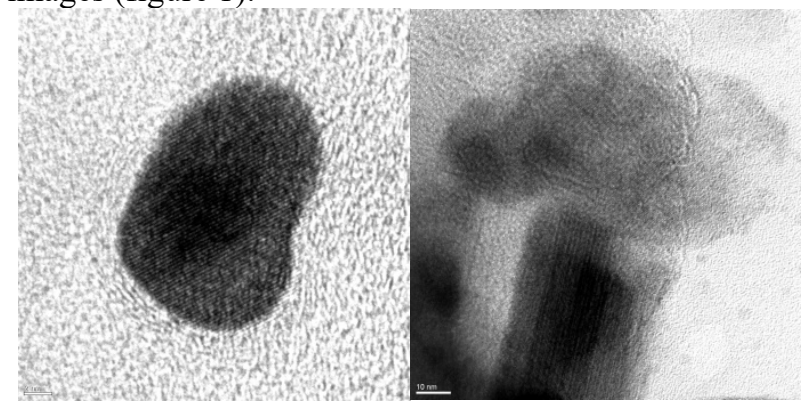

Figure 1. Examples of images shoot in direct resolution mode

The results were compared with the results of calculations of specialized software (Gatan Microscopy Suite v.1.8) [7]. An example of image processing using this software is presented in figure 2 .

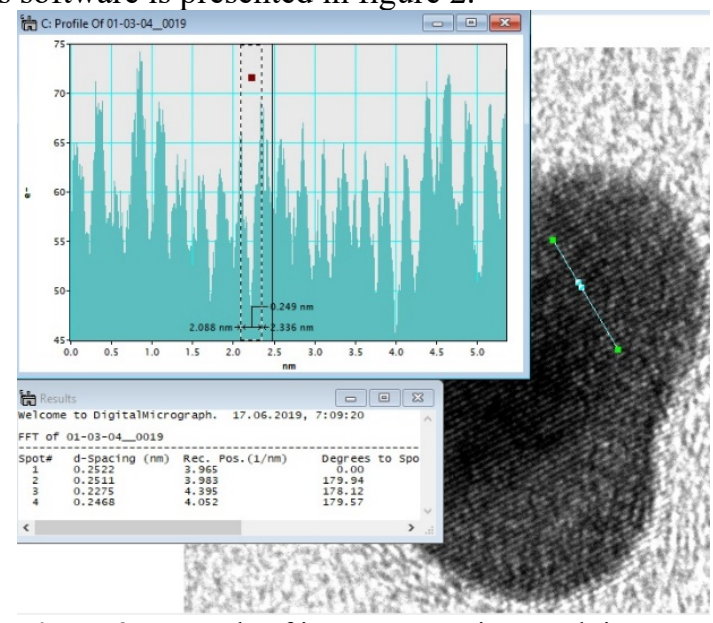

Figure 2. Example of image processing result in Gatan Microscopy Suite v.1.8
As a result of comparing the values obtained in the framework of the developed algorithm with the values obtained by specialized software and used as reference, insignificant discrepancies of the order of $0.005 \mathrm{~nm}$ were obtained, which indicates the successful development of an analogue to existing algorithms.

\section{Determination of parameters of the crystal lattice of materials}

Within the framework of increasing the level of automation of TEM algorithms, determining of the crystal lattice parameters of material samples was chosen as the next task.

The phase composition and orientation of the crystals can be determined from the diffraction patterns of the samples.

Diffraction contrast is formed as a result of elastic scattering of transmitted electrons by regularly located atoms in the crystal lattice.

Contrast is used to determine the crystallinity of the film and the lattice parameter (figure 3 ).

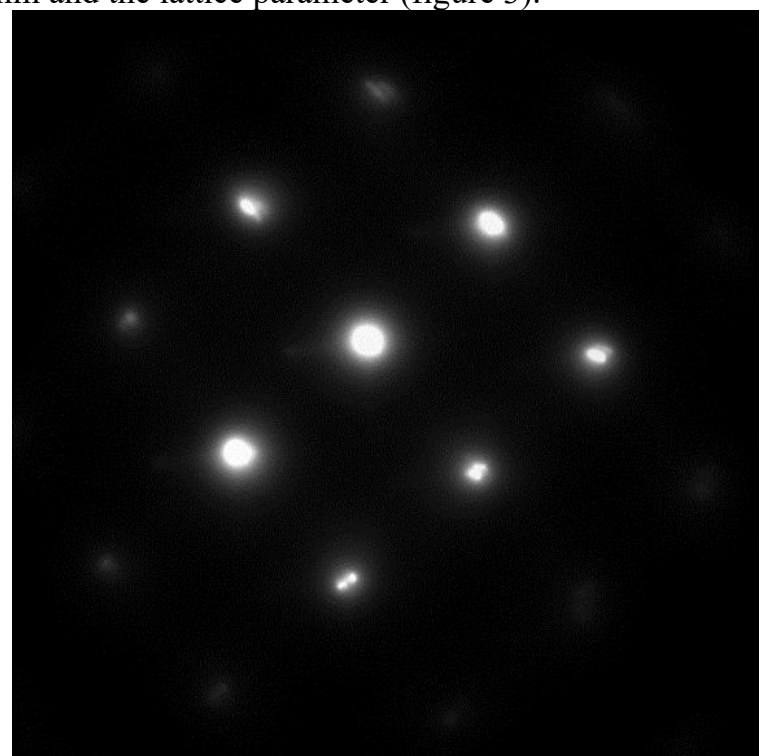

Figure 3. Example of a diffraction pattern of a silicon lattice

Specialized software processes these images in a semiautomatic mode, requiring the selection of starting points for calculations. An example of such processing is presented in Figure 4 [6]

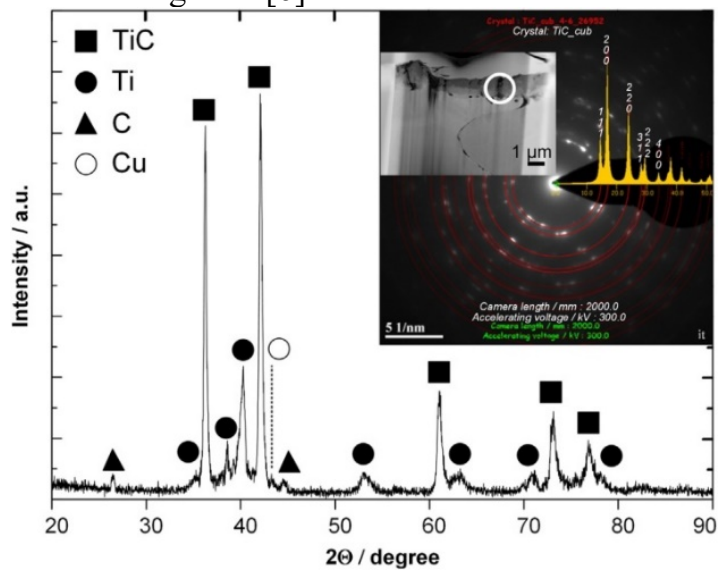

Figure 4. An example of processing a diffraction pattern by specialized software 
As can be seen from figure 4, the software function consists of determination of brightness in a circular radius from the center of the diffraction pattern, as well as calculation of the number of points on concentric circles and the distance between these circles, which characterize the material sample. The problem with this approach is in the necessity of accurate manual selection of the center of the image and the distance to one of the concentric circles, consisting of bright points.

In addition, not all images of the diffraction pattern have the same high quality, which allows to determine important characteristics. Images with excessive brightness of the central area can be the most frequent example of low quality images (Figure 5). The situation with reflection of points is also often can be found (Figure 6). These specific cases make difficulties in developing a universal automatic algorithm that would be able to parse all particular cases with the same efficiency.

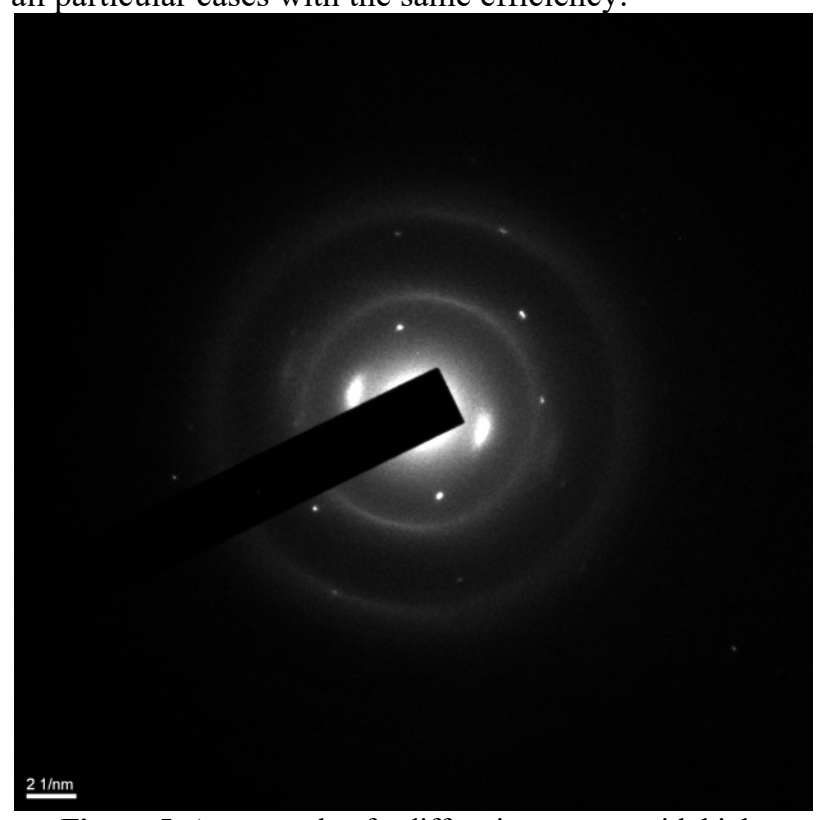

Figure 5. An example of a diffraction pattern with highbrightness area in the center of an image

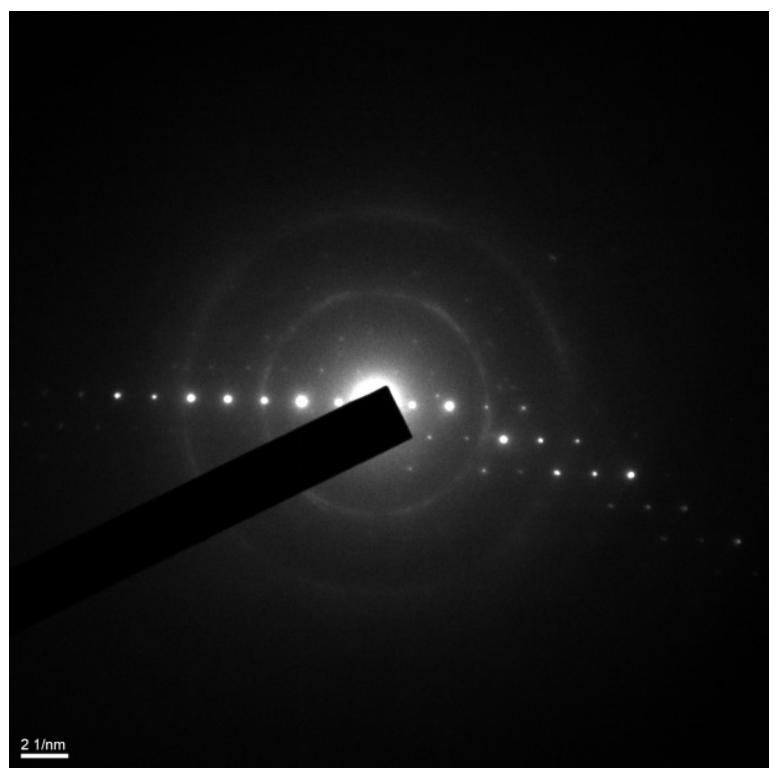

Figure 6. An example of a diffraction pattern with reflection of points

\section{Evaluation of the effectiveness of image processing algorithms in the task of automating the determination of crystal lattice parameters}

There are a number of algorithms that can be useful in automating the processing of diffraction patterns. A number of well-known image processing algorithms and the possibilities of its applying to this task were considered.

For images with a sufficiently high contrast, the determination of distances can be carried out by transferring the entire image to the frequency domain, for example, using a variation of the Fourier transform (DFT). However, in case of irregular brightness or if the image does not fit the conditions for such a conversion, it will be impossible to determine anything from it.

The Watershed method of automatic image segmentation $[10,11]$, available in the OpenCV library of computer vision algorithms, was considered as one of the possible directions in the automation of determining the number and location of bright points in the image of the diffraction pattern. An example of his work for such images is presented in Figure 7.

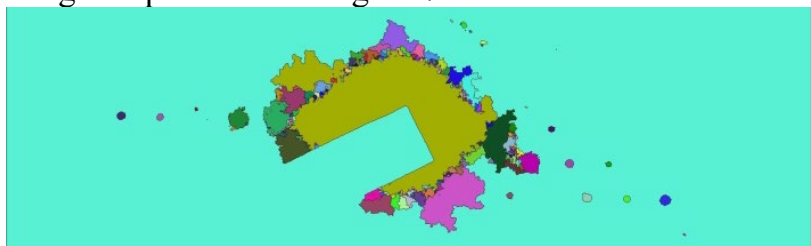

Figure 7. An example of image segmentation using the Watershed algorithm

The advantages of using this algorithm are in automatic selection of all areas with large differences in the brightness of the image and in determination of their size and position. The disadvantage of this algorithm is that it is very sensitive to the choice of the brightness threshold and to its irregular distribution in the processed image. Figure 7 shows that, despite the successful selection of points remote from the center, the entire area in the center almost merges into one continuous segment.

Given the specifics of the images of the diffraction pattern of materials, it can be assumed that a modification of this algorithm that dynamically changes the sensitivity threshold from the center of the image to its borders can become quite effective for the task of evaluating these images.

Another, simpler automation option may be the calculation of a binary image according to a similar principle, with a dynamic sensitivity threshold, taking into account the decrease in brightness of images from the center to the borders (Figure 8).

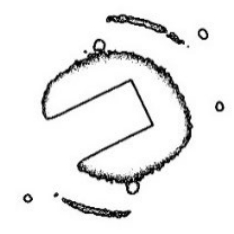

Figure 8. Example of a binary image of a diffraction pattern 
Thus, it can be assumed that for the task of determining the characteristics of the diffraction pattern, the most effective direction will be a complex algorithm combining binarization and segmentation to automate the search for the image center and bright points distant from it with the algorithm for calculating the brightness of concentric circles traditional for specialized software with previously known positions of the center and distance to these circles.

Further work in automation of the processing of images of diffraction patterns will be carried out in the direction of developing specific modifications of the considered algorithms.

\section{Results}

The review of the existing approach to processing images of the crystal structure of materials using specialized software for analyzing TEM data is carried out.

The analysis of the problem of estimating the parameters of the crystal structure from the image of the diffraction pattern of a material sample is carried out; image processing methods suitable for automating this task are identified. A number of possible modifications of existing algorithms are proposed, which allow to process such images in a partially automatic mode.

The development of these modifications will make it able to solve the problem of estimating the parameters of the crystal structure without involving specialized software.

\section{Acknowledgments}

The work was supported by RFBR, Grant № 18-41700001 .

\section{References:}

[1] Leutenegger S. BRISK: Binary Robust invariant scalable keypoints / S. Leutenegger, M. Chli, R.Y. Siegwart // Proceedings of the 2011 International Conference on Computer Vision (ICCV '11). 6 November 2011. P. 2548-2555.

[2] Nebaba S.G. An Algorithm for Building Deformable 3d Human Face Models and Justification of its Applicability for Recognition Systems / S.G. Nebaba, A.A. Zakharova // SPIIRAS Proceedings. 2017. Vol. 52. P. 157-179.

[3] Schettini R. Underwater Image Processing: State of the Art of Restoration and Image Enhancement Methods / R. Schettini, S. Corchs // EURASIP Journal on Advances in Signal Processing. 2010.

[4] Sokratis V. A Hybrid Binarization Technique for Document Images / V. Sokratis, E. Kavallieratou, R. Paredes, K. Sotiropoulos // In: Biba M., Xhafa F. (eds) Learning Structure and Schemas from Documents. Studies in Computational Intelligence. 2011. Vol. 375. Springer, Berlin, Heidelberg.

[5] Alhadidi B. Mammogram Breast Cancer Image Detection Using Image Processing Functions / B. Alhadidi, M.H. Zu'bi, H.N. Suleiman. // Information Technology Journal. 2007. Vol. 6. №. 2. P. 217-221.

[6] Sivkov, A. Deposition of a TiC/Ti coating with a strong substrate adhesion using a high-speed plasma jet / Sivkov, A., Shanenkov, I., Pak, A., Gerasimov,
D., Shanenkova, Y. // Surface and Coatings Technology. 2011. Vol. 291. P. 1-6.

[7] Gatan Microscopy Suite Software [Electronic Source]. URL: https://www.gatan.com/products/temanalysis/gatan-microscopy-suite-software. (Last accessed: 11.06.2019).

[8] Nebaba S.G., Pak A.Y., Zakharova A.A. Automated Algorithm for Determining the Interplanar Distances of the Crystal Structure of a Substance from Transmission Electron Microscopy Images // CEUR Workshop Proceedings. 2019. Vol. 2485. pp. 248251. DOI: $10.30987 /$ graphicon-2019-2-248-251

[9] Gonzalez R.C. Digital Image Processing (3rd Edition) / R.C. Gonzalez, R.E. Woods // Prentice-Hall, Inc., Upper Saddle River, NJ, USA, 2006. P. 976.

[10]Zaripova A.D., Zaripov D.K., Usachev A.E. Visualization of high-voltage insulators defects on infrared images using computer vision methods // Scientific Visualization. 2019. Vol. 11 (2). pp. 88-98.

[11] Khvostikov A.V., Krylov A.S., Mikhailov I.A., Malkov P.G. Trainable active contour model for histological image segmentation // Scientific Visualization. 2019. Vol. 11 (3). pp. 80-91.

\section{About the authors}

Nebaba Stepan G., Ph.D., engineer of Division for Automation and Robotics of Tomsk Polytechnic University. Email: stepan-lfx@mail.ru.

Pak Alexander Ya., Ph.D., associate professor of Division for Automation and Robotics of Tomsk Polytechnic University. Email: ayapak@tpu.ru. 